\title{
PROCESOS DE POBLAMIENTO, INTEGRACIÓN Y MARGINALIDAD EN UN TERRITORIO PERIFÉRICO DE LA PATAGONIA MERIDIONAL. EL CASO DE LOS ANTIGUOS
}

MARÍA EUGENIA CEPPARO DE GROSSO*

\section{RESUMEN}

El área de estudio es el sector noroeste de la provincia de Santa Cruz, más precisamente la localidad de Los Antiguos. Se considera la función que este asentamiento desempeñó en la subregión patagónica meridional a lo largo de su corta historia; y el desarrollo de la agricultura en el marco de un territorio marginal, con limitaciones ambientales y tradicionalmente pastoril. Estos temas se profundizan según los procesos de ocupación e integración en un área distante y con problemas de accesibilidad; y de crecimiento de la población y su orientación a las actividades terciarias acompañando la tendencia del modelo provincial.

PALABRAS CLAVES: Patagonia Meridional, Los Antiguos, poblamiento, integración, marginalidad.

\section{INHABITATION, INTEGRATION AND MARGINALIZATION MECHANISMS IN THE PERIPHERAL TERRITORY OF SOUTHERN PATAGONIA. THE CASE OF LOS ANTIGUOS}

\section{ABSTRACT}

The field site discussed in this paper is in the northwest of the province of Santa Cruz, more specifically, the locality of Los Antiguos. The paper seeks to study the village's role in the meridian sub-region of Patagonia. It also focuses on the development of agriculture within the context of a typically pastoral and marginal territory that has suffered negative environmental impacts. These issues are discussed in the light of this region's inhabitation and integration mechanisms, population increase, and the tendency to engage in tertiary activities - thus reproducing the 'provincial model'.

KEYWORDS: Southern Patagonia, Los Antiguos, inhabitation, integration, marginalization

* Departamento de Geografía, Facultad de Filosofía y Letras, Universidad Nacional de Cuyo - Consejo Nacional de Investigaciones Científicas y Técnicas (CONICET). mcepparo@logos.uncu.edu.ar 


\section{INTRODUCCIÓN}

En este artículo se analiza el proceso de poblamiento, la ocupación del territorio y el crecimiento de la población en el noroeste de la provincia de Santa Cruz, en la Patagonia meridional argentina, precisamente en Los Antiguos, desde comienzos del siglo XX hasta nuestros días. EI marco teórico, sobre el que se apoyan los hechos, se basa en los conceptos de procesos y marginalidad. El primero, porque involucra las ideas de cambios, avances, retrocesos, estancamientos, que obedecen a hechos coyunturales 0 estructurales regionales y nacionales y que afectan especialmente a zonas vulnerables como la de este estudio. Es por eso que se vinculan estos procesos con las características intrínsecas de las regiones marginales y con el hecho paradojal que experimentan algunas zonas consideradas como tales, pero con funciones claves en la integración y en la vinculación con territorios vecinos; y entre ellos y los espacios extralocales.

Se considerará la función que Los Antiguos desempeñó en la subregión patagónica meridional a lo largo de su corta historia; y el desarrollo de la agricultura en el marco de un territorio marginal, con limitaciones ambientales y tradicionalmente pastoril. Estos temas se profundizan según: 1 , los procesos de ocupación de la zona noroeste de Santa Cruz y la integración entre Los Antiguos y Chile Chico en la Región de Aysén. Ambas localidades afectadas por las distancias y por problemas de accesibilidad; y 2 , los procesos de crecimiento de la población de LoS Antiguos y su orientación a las actividades terciarias, acompañando la tendencia del modelo provincial.

Metodológicamente, los trabajos personales en la zona, las encuestas y entrevistas con detenimiento, además de la selección y evaluación crítica de los documentos relacionados con el espacio y los hechos, constituyeron procedimientos claves para lograr la aproximación a las conclusiones.

1. LOS PROCESOS Y LOS CAMBIOS. LAS ÁREAS MARGINALES Y LOS CRITERIOS DE DEFINICIÓN

Los procesos pueden considerarse desde dos puntos de vista: desde el espacio y desde el tiempo. Desde la visión de las características espaciales se destacan las diferencias de las apti- tudes de los elementos ambientales y la ubicación de sus posibilidades. Otra propiedad de los objetos geográficos se refiere a la sucesión de los hechos, es decir las modificaciones que los lugares han experimentado en el tiempo y que contribuyen con el conocimiento de su evolución y dinámica actual. Interesa la secuencia de los procesos porque le dan a un sistema su carácter articulador o por el contrario provocan la carencia de objetivos unificadores. A través del estudio de los procesos, y con el auxilio de la historia, se identifican, los ciclos de crecimiento poblacional y económico, las causas de los avances y retrocesos en el desarrollo de las actividades, los cambios de funciones de los territorios y las fuerzas que actúan sobre esos cambios de estado y los controlan. Cada secuencia temporal coincide con una variación de estructura y de organización espacial, a veces profunda, a veces leve, notoria en el corto y en el largo plazo ${ }^{1}$.

Los cambios son visibles en el espacio, especialmente cuando se relacionan con las actividades humanas. Son la manifestación de un gran número de decisiones pasadas y presentes. K. Chapman analizó intensamente la influencia de los procesos de decisión en la localización de las actividades y en el uso de la tierra. Según su opinión las decisiones de individuos o grupos de individuos no se hacen en el vacío, están fuertemente influenciados por el medio ambiente dentro del cual opera el que toma la decisión. ... . De todos modos es inaceptable la filosofía determinista del medio físico sobre el humano. . . El espacio terrestre no sólo contiene los atributos del medio físico, sino también los rasgos hechos por el hombre que reflejan una fuerte interrelación entre las distribuciones de las actividades humanas del pasado, presente y futuro? ${ }^{2}$.

Distribuciones que indican diferentes pautas de poblamiento y desarrollo económico que pueden provocar una retroalimentación negativa o positiva del sistema, es decir pueden generar situaciones diferentes a las previstas originalmente. Las motivaciones ventajosas iniciales pueden haber generado, con el tiempo, tensiones inesperadas difíciles de solucionar. De allí que la determinación de la continuidad o discontinuidad de los procesos que marcaron el manejo del territorio, también conlleva reflexionar so-

\footnotetext{
Gutiérrez y Furlani 1993, op. cit., p.96

2 Chapman 1979, op. cit., pp. 12-13.
} 
bre la valoración que los actores han hecho de sus rasgos locales: físicos, sociales, políticos y culturales. Además, la consideración de la evolución del componente social, permite supera la visión desde lo físico, como tradicionalmente se ha realizado en la explicación de la dinámica de un territorio extenso y limitante como el de la Patagonia Meridional.

Superación que también se apoya en los conceptos de regiones marginales, territorios periféricos, remotos, escasamente poblados, entre otras denominaciones, no siempre equivalentes. De allí que considero necesario hacer un rápido recorrido por las ideas de algunos estudiosos del tema. Consideraciones que alimentan el marco teórico sobre el que se basa este trabajo.

Existe una generalizada orientación de los institutos de investigación geográfica, de grupos interdisciplinarios y, particularmente, de los geógrafos hacia los estudios de las áreas de fuerte concentración urbana, industrial y de gran intensidad en el uso agrario o con tradición agrícola. Las complejas estructuras productivas, la intensa inversión de capitales y tecnología y la fuerte atracción de mano de obra, han incentivado la inclinación de los estudios hacia esos fenómenos y esos espacios. Por el contrario, son pocos los trabajos relacionados con las áreas rurales que encierran dispersas y escasas poblaciones dedicadas a emergentes cultivos y más aún si éstas se ubican en territorios como el que es objeto de este estudio. Apreciación que surge luego del análisis de las publicaciones de centros de investigación internacionales y nacionales dedicados a esos temas.

En el marco internacional, y según los estudios realizados desde 1992 por el Grupo de Estudios sobre Problemas de Desarrollo en Áreas Marginales de la Unión G eográfica Internacional (UGI), se observa el interés creciente de los geógrafos por investigar, entre otros temas: el impacto del avance tecnológico y de la reorganización social en áreas marginales, sus políticas y estrategias; la sustentabilidad económica y social en esos territorios; las percepciones regionales de la marginalización; la globalización y la marginación; los problemas de la marginalidad y la agricultura.

Uno de los especialistas, W. Leimgruber, señala los motivos de esta tendencia: problemas económicos y sociales han aumentado el desarrollo de las regiones marginales en los países desarrollados y en vías de desarrollo. Al mismo tiempo, asisti- mos a un incremento de la marginación de las regiones y de los grupos humanos debido a la continua presión sobre las tierras y los recursos, la tendencia al deterioro de la economía mundial y el aumento de las estructuras del poder central ${ }^{3}$.

Leimgruber intenta clarificar el concepto de marginalidad, pero reconoce que existe una amplitud de criterios -geométrico, ecológico, económico, social, cultural, político y aún el percibido-, que hacen difícil estudiarla desde un punto de vista específico o desde una sola escala 4 . Por el contrario es necesario abordar el concepto desde variadas visiones complementarias ${ }^{5}$. Esa combinación de perspectivas provoca, en ocasiones, situaciones paradójicas. Es el caso de la idea generalizada que las regiones periféricas o alejadas también son marginales, sin embargo, Leimgruber aclara que el aislamiento o la dificultad en la movilidad entre los centros no siempre corresponde con la marginalidad de las sociedades ${ }^{6}$.

La situación se agrava cuando se suman limitados equipamientos, escasa disponibilidad de oportunidades, cerrado ambiente social y cultural, y falta de capacidad de innovación de los propios actores sociales. Situación que permite identificar la realidad de esas regiones con el modelo desarrollado por Andreoli y Tellarini con respecto a la ubicación de las áreas marginales en el extremo final del continuo regional centro-periferia. $Y$ seguramente en ellas el futuro socio-económico-cultural es más sombrío, frágil y vulnerable que en la periferia7.

La misma noción de marginalidad, tan amplia y compleja por sus múltiples relaciones, hace que la transferencia de los resultados desde los países o instituciones que más tratan el tema no sea tan fácil de realizar, ni tampoco adecuada debido a que predominan los trabajos con la perspectiva de los investigadores de los países más representados en ese Grupo de Trabajo, los países centrales de Europa y América del Norte. Por lo tanto, aportan una parcializada visión conceptual y metodológica. De hecho, de la lectura de las Actas de las Reuniones, comprobé que solamente un promedio del $25 \%$ de los trabajos presentados se refiere a problemas de marginalidad

3 Leimgruber 1994, op. cit., p. 1.

4 Ibid. p. 8.

5 Schmidt 1998, op. cit., p. 271.

6 Leimgruber 1994, op. cit, pp.1-8.

7 Andreoli, Tellarini et al 1989. op. cit., p. 12 
en países subdesarrollados o del Tercer Mundo ${ }^{8}$

La referencia al trabajo de estas comisiones o grupos de investigación, demuestra que aún en países desarrollados y con una importante cultura industrial, el problema de la marginalidad está vigente e involucra temáticas muy variadas. Con más razón se considera oportuno intensificar el estudio de esas cuestiones y de los espacios afectados en países en vías de desarroIlo, subdesarrollados o subindustrializados, en regiones aisladas, en territorios vulnerables.

\section{LOS ENTORNOS TERRITORIALES Y SOCIO- ECONÓMICOS DE SANTA CRUZ}

Santa Cruz es la provincia más austral del territorio continental argentino, la segunda en extensión y una de las más despobladas de la República Argentina. Las características climáticas, frías áridas, y su gran tamaño, 240.000 $\mathrm{km}^{2}$, anticipan sus rasgos físico-bióticos extremos y la convierten en un lugar difícil de poblar y explotar económicamente. Desde cualquier lugar donde se inicie el recorrido por la provincia, ya sea desde la costa hacia el interior, por la ruta provincial № 43 hasta Los Antiguos; o a lo largo de la ruta nacional № 3 hasta llegar a Río Gallegos, aparecen elementos constantes en el paisaje. La imponencia del mismo está dada por la monocromía y regularidad de las formas mesetarias que se suceden sin interrupción, teniendo de fondo la infinita visión del horizonte sin que se interponga ningún obstáculo. Las formas tabulares del relieve, están atravesadas por cauces secos y anchos valles encajonados. Existe un fantástico desarrollo de infinidad de cañadones de variada amplitud que conforman un majestuoso panorama.

8 En América Latina existen organismos de planificación que se ocupan de los problemas que caracterizan a las regiones críticas y marginales. Investigadores como Patricia Wilson de SIAP (Sociedad Interamericana de Planificación); F. Alburquerque Llorens de ILPES (Instituto Latinoamericano y del Caribe de Planificación Económica y Social), J. Rocatagliata, H. Bozzano y J . Coraggio de Argentina; Sergio Boisier y $C$. de Mattos de Chile; y Friedmann de Estados Unidos, entre otros, tienen numerosos trabajos publicados, sobre todo, en la Revista Interamericana de Planificación de la SIAP. Es necesario destacar la labor de los planificadores preocupados por un cambio conceptual, metodológico y técnico para abordar y solucionar distintas situaciones que afectan a países escasamente poblados o marginales de América Latina.
Pequeños y medianos asentamientos muy aislados entre sí, con evidentes signos de ser centros de servicios para las áreas rurales vecinas, aparecen y desaparecen de repente en los caminos. Caminos que, por otra parte, se caracterizan por sus recorridos lineales sin grandes inflexiones, que favorecen la visión panorámica y el contraste de colores entre la vegetación verde-grisáceo o pardusca de los suelos y los azulesturquesas del cielo. En algunos casos, la presencia de forestación anuncia la llegada de alguna de las poblaciones o indican, desde lejos, la ubicación de los establecimientos ganaderos. Éstos se localizan generalmente lejos de las rutas y protegidos por las barrancas de las mesetas.

El impacto que provoca la persistencia y la violencia de los vientos secos que vienen del oeste, acrecienta la sensación de la austeridad del medio. Austeridad que también genera la visión de la continua y rala vegetación natural, de tipo estepario, que crece sobre suelos cubiertos por cantos rodados. Climáticamente, el área se percibe fría y árida. Las lluvias invernales, las nevadas o las escarchas perjudican el estado de los caminos y la regularidad del tránsito, dejan a las poblaciones bloqueadas durante días; o afectan la actividad agropecuaria ya que impiden el retorno de los rebaños a los lugares más protegidos. La incomunicación es sentida con más intensidad y frecuencia en el ámbito rural. Los pobladores experimentan los inconvenientes que genera la distancia a la costa, a las vías de comunicación en buen estado, a los centros más abastecidos. Ausencias que, en verdad, fortalecen 0 retraen el espíritu.

A lo largo de todos los itinerarios, predomina el uso ganadero del suelo que no manifiesta signos de dinamismo o modificación. La desertización y el mal uso de los campos son evidentes por los extensos suelos desnudos que se repiten en todos los trayectos. Las majadas son guiadas por solitarios peones a caballo acompañados por los perros ovejeros; y pocas veces se observa otro tipo de actividad que represente acciones sistemáticas para mejorar los suelos 0 para producir forrajes.

Por otra parte, a lo largo de la corta y lenta ocupación del territorio santacruceño, hace poco más de un siglo, el manejo del ovino dominó el espacio rural. Los antecedentes culturales de las primeras ondas de poblamiento, se sumaron a las limitaciones del ambiente para conducir la explotación económica a la ganadería muy 
extensiva. Desde la primera mitad del siglo $\mathrm{XX}$, la explotación de hidrocarburos y carbón no provocó otras actividades más allá de las extracciones. Más cerca en el tiempo, la pesca dinamizó algunos puertos. Mientras tanto el territorio se fue organizando, desde el punto de vista urbano, sobre la base de una periférica red de ciudades articuladas por una elemental infraestructura con debilidad en la conectividad, accesibilidad y densidad de las rutas ${ }^{9}$. La situación que predomina en las ciudades es la concentración de las actividades terciarias, con la administración pública en primer lugar.

Los impactos territoriales más destacados los produjo la ganadería. El mal uso de los pastos, provocado por el sobrepastoreo acrecentó la tendencia a la desertización que el medio por sí solo generaba. Una larga secuencia de factores coyunturales y estructurales relacionados con la actividad agropecuaria fueron generando sucesivas crisis económicas. La disminución de la cantidad de cabezas de ganado y del volumen

9 Cepparo 1992, op. cit., pp. 77-90 y Civit et al 1991, op. cit., pp. 167-201. de lana producida, fueron las principales consecuencias. La agricultura siempre estuvo escasamente representada y limitada a sitios muy específicos. Algunos establecimientos ganaderos cultivan forraje, pero todavía continúan dependiendo de los pastos naturales. Y en la mayoría se cultiva hortalizas y frutales al aire libre o bajo invernadero.

A pesar de las acentuadas condiciones de aridez, existen sitios favorecidos por la mayor cantidad de precipitaciones, por el caudal de los ríos, por microclimas especiales o por el empeño de algunos productores. Ya sea como cultivos en secano o bajo sistemas de riego con diferente grado de organización, han surgido y crecido pequeñas áreas de producción agrícola intensiva. Se cultiva frutas finas, especialmente cerezas, en Los Antiguos, con un incipiente mercado externo; variedades de hortalizas de hoja con rápida colocación en el mercado local, en Río Gallegos; y en Gobernador Gregores, cultivos de alfalfa y ajo colorado santacruceño. Estas reducidas plantaciones de cerezas, hortalizas y alfalfa en los extremos noroeste, sureste y centro de la provincia, han roto la monotonía de sus actividades rurales, y son una perspectiva de cambio económico

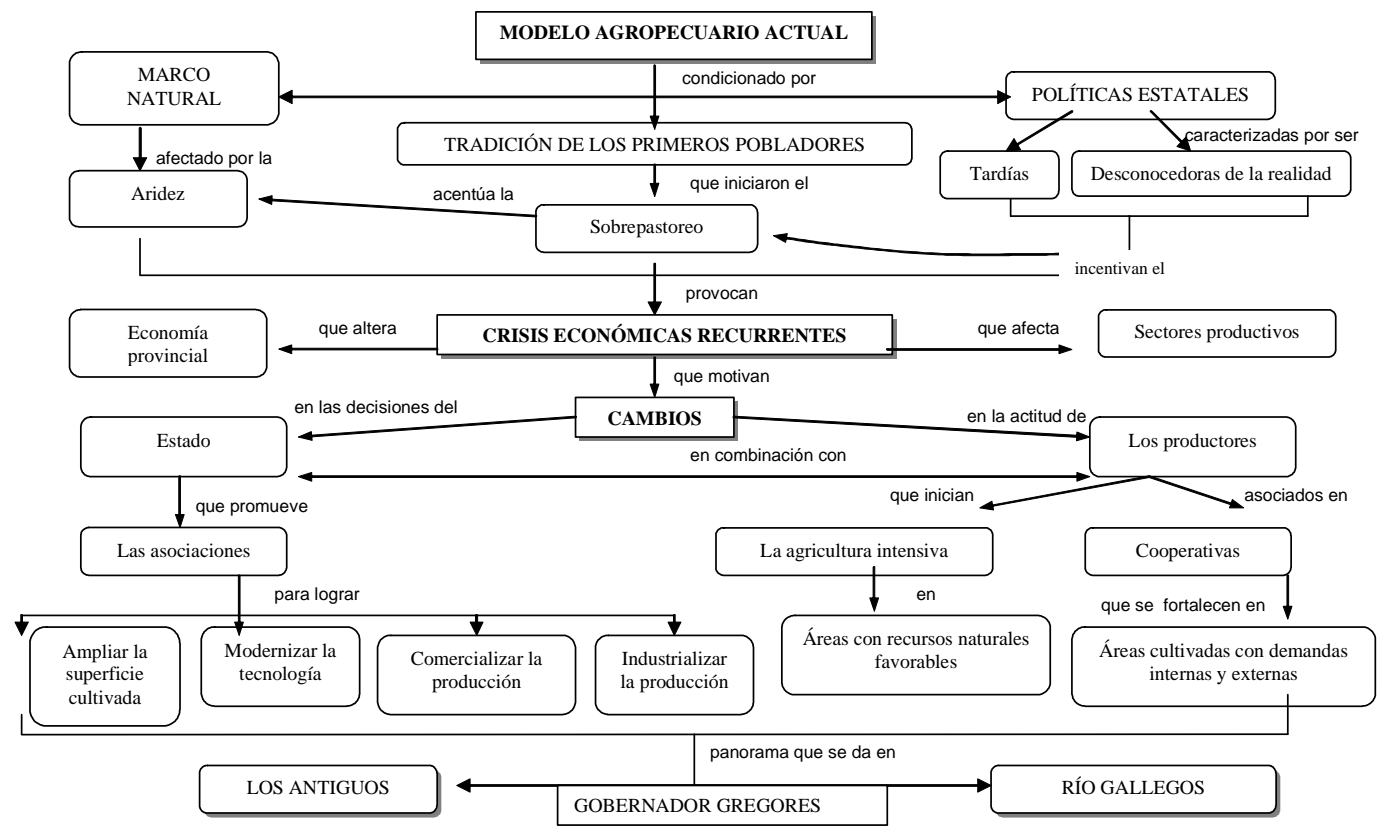

Fig. 1 Santa Cruz. Un modelo conceptual de lo agropecuario. Crisis y motivaciones para el cambio. Fuente: Elaborado por María Eugenia Cepparo sobre la base de la bibliografía consignada y trabajo de campo. 
por los impactos socio-territoriales que pueden provocar. La figura 1 constituye un modelo conceptual referido a los procesos de crisis y transformaciones experimentadas por la actividad agropecuaria actual en Santa Cruz.

\section{SIGNOS DE DINAMISMO EN EL EXTREMO NOROESTE DE LA PROVINCIA}

La monotonía que caracteriza el ámbito rural santacruceño se interrumpe solamente en en las aisladas manchas de explotación minera como sucede en los tramos entre Comodoro Rivadavia-Caleta Olivia-Las Heras; 0 de explotación forestal y agrícola en Perito Moreno y Los Antiguos; y en los dispersos puertos pesqueros que coinciden con las ciudades costeras, unidas por la ruta nacional pavimentada № 3 .

La fisonomía del área urbana y de las zonas de cultivos de la localidad de Los Antiguos no se asemeja a las demás. Mucho antes de llegar a esta localidad del noroeste provincial, se aprecia una abundante vegetación. Distintos tonos de verde y ocre, de plantaciones de forrajeras, de cortinas de álamos perpendiculares al camino y de frutales, preanuncian un panorama rural dis- tinto. Ese conjunto de vegetación provoca que la llegada a Los Antiguos sea sorprendente y muy atractiva. La pequeña ciudad se extiende por la calle principal, continuación de la ruta provincial pavimentada $\mathrm{N}$ ㅇ 43. Se localiza en la margen sur del lago Buenos Aires, a 986 km de Río Gallegos y a $360 \mathrm{~km}$ de Caleta Olivia, comunicada por dos líneas de transporte de pasajeros, que llegan a Los Antiguos dos veces por día desde la costa. Otras empresas los trasladan hasta Chile Chico en territorio chileno, desde donde recorren las regiones de Aysén y Magallanes.

El hospital, la pintoresca municipalidad, almacenes de ramos generales, los dos hoteles, la Cooperativa Frutícola El Oasis, y la nueva Empresa Empacadora Río Alara S.A., se ubican sobre la calle principal o muy próxima a ella. Desde aquí y hacia el norte y el sur se extiende el área urbana a lo largo de pocas cuadras, diluyéndose entre las parcelas dedicadas, especialmente, al cultivo de frutales de carozo. La mancha intensamente cultivada está alimentada por una red de riego que nace de los ríos J einimeni y Los Antiguos (Fig. 2). Canales y acequias con agua permanente acompañan las vías de acceso a la localidad y los caminos internos.

Ya en el interior del espacio cultivado, el

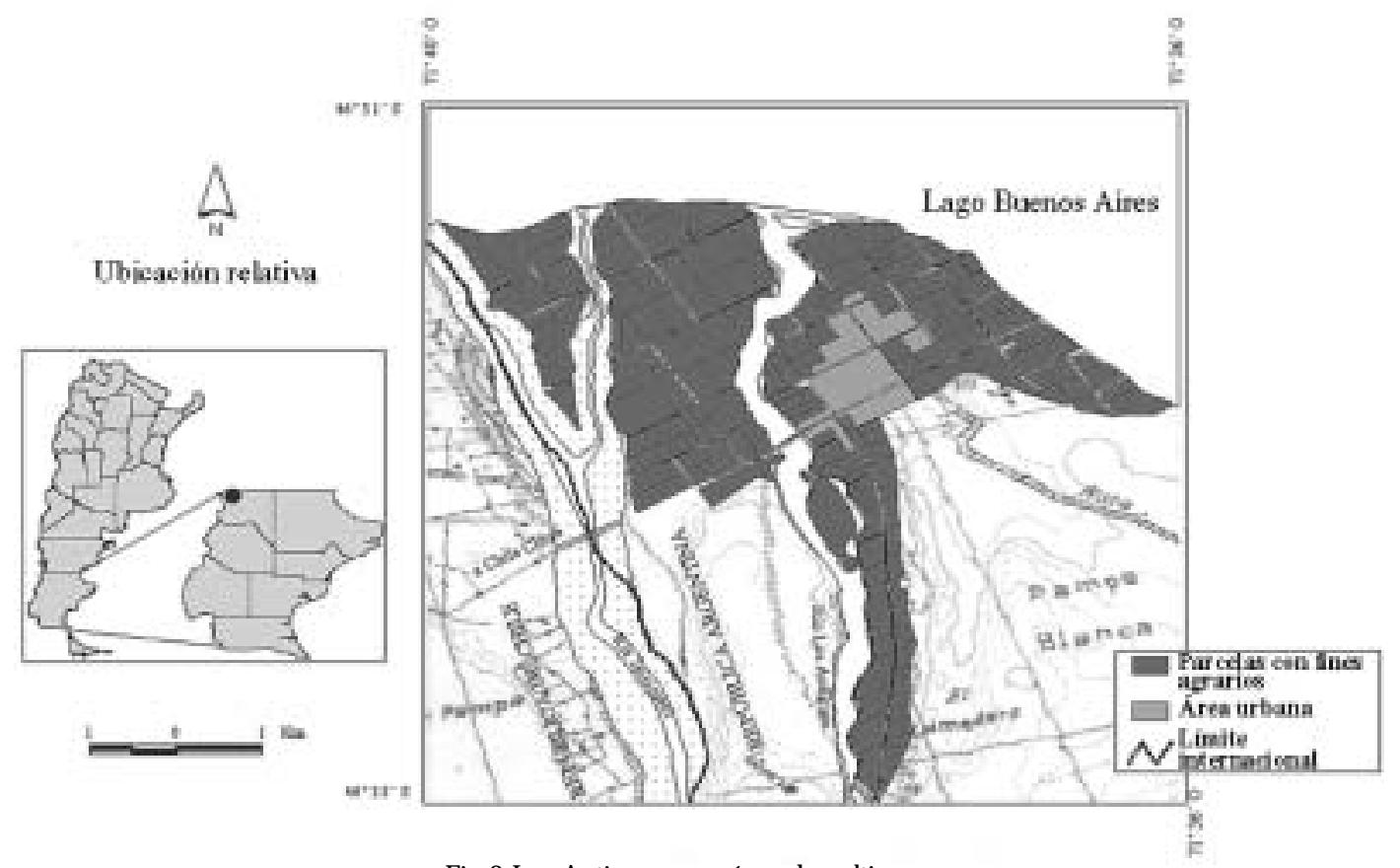

Fig.2 Los Antiguos y su área de cultivo.

Fuente: Elaborado por M.E. Cepparo sobre la base del plano "Lotes agrícolas de la Colonia de los Antiguos". Municipalidad de Los Antiguos, Santa Cruz. 1999; y carta topográfica LOS ANTIGUOS №4772-10. Escala 1:100.000. IGM 
parcelamiento experimenta modificaciones en cuanto a su estado, disposición y tamaño. Parcelas sin plantaciones cortan con frecuencia los espacios vigorosamente ocupados por cultivos al aire libre, la mayoría de las veces ocultos por las densas cortinas de álamos que también resguardan las viviendas y construcciones anexas que varían de dimensiones, número y calidad según la capacidad económica de los propietarios. Cerezas, ciruelas, duraznos, frutillas, grosellas y otras frutas rojas son las preferidas, aunque las primeras predominan.

Sólo en los últimos diez años, los pobladores de la localidad de Los Antiguos -2.047 habitantes según el censo de 2001-y aproximadamente a $2.500 \mathrm{~km}$ al sur de la ciudad de Buenos Aires, han comenzado a obtener muy buenos resultados en las cosechas de cerezas y en las ventas en el mercado externo. La fruticultura del valle, de aproximadamente 1.300 ha, se ha extendido y modernizado; y los parámetros de calidad han pasado a tener un rol fundamental en el comercio internacional. La explotación agraria, la forestación con cortinas de álamos, el sistema de riego y los primeros tratamientos de la fruta, se han difundido rápidamente; y la población ha comenzado a valorizar la agricultura y a comprometerse con todos los eslabones de la cadena agroalimentaria de la cereza. Las plantaciones ocupan un reducido espacio y los predios intensamente ocupados por frutales de carozo se intercalan con dilatadas superficies to davía sin cultivar. Sin embargo, los trabajos en el terreno permitieron detectar una estructura agraria cada vez más compleja, y a pesar de la micro escala en la que suceden los hechos, el sistema socio-territorial agrario que se está formando, experimenta los mismos procesos que otros sistemas más afianzados, con más tradición y mejor localizados en la región centro-sur del país ${ }^{10}$.

4. EL PROCESO DE POBLAMIENTO Y EL INICIO DEL DESARROLLO ECONÓMICO EN EL MARCO DE LA POSICIÓN FRONTERIZA Y MARGINAL

\subsection{Los comienzos del poblamiento}

Es cierto que el norte de la provincia de Santa Cruz o Zona del Río Deseado, como tradicionalmente se la llamaba a fines del siglo XIX,

10 Cepparo, 2000, 2005, op. cit. fue la que recién en la década de 1920 se ocupó con pobladores que continuaron con la orientación económica del resto del territorio: la ganadería ovina. No obstante, no significa que no haya sido motivo de interés por parte de la Dirección de Tierras. El presidente Irigoyen se apoyó en la Ley 4.167 del 8 de enero de 1903 para autorizar ...al Poder Ejecutivo a explorar y medir tierras fiscales y una vez cumplido este requisito determinar el destino de las distintas zonas... reservando las regiones que resulten apropiadas para la fundación de los pueblos y establecimientos de colonias agrícolas; y en la Ley 5.559 de Fomento de Territorios Nacionales... que distinguía las coIonias agrícolas de las ganaderas y establecía la manera de poblarlas. ${ }^{11}$

A partir de ese marco legal, se crearon una serie de colonias pastoriles en el norte y oeste de Santa Cruz con el fin de atraer pobladores. Estas colonias, con una superficie aproximada de 5.000 ha, fueron: Las Heras en el centro-norte de la provincia sobre el río Deseado; Presidente Manuel Quintana y Presidente Carlos Pellegrini en las proximidades del lago Posadas y lago Pueyrredón; Presidente Luis Saenz Peña y General Paz en las nacientes del río Chico en el centro-oeste. Unos años más tarde, por el decreto nacional del 11 de junio de 1921, se establecieron las colonias pastoriles de L eandro N. Alem al sur del lago Buenos Aires, en la denominada Zona del Río Deseado y en el noroeste de la provincia; y Francisco P. Moreno al sur del lago Argentino, en el suroeste ${ }^{12}$.

También en Chile, desde los inicios del siglo XX, y como resultado de la misma inquietud por ocupar el territorio, incorporarlo a la economía del país y demostrar la soberanía en la Patagonia, el presidente Germán Riesco decide, basado en la ley del 4 de agosto de 1874, llamar a licitación de arrendamiento de las tierras ubicadas al sur del lago General Carrera, ex lago Buenos Aires, llamado en ese momento Territorio de Aysén. La legislación favoreció, ya sea a pobladores libres que intentaron afincarse en la zona, como también a importantes hombres de empresa de Magallanes que adquirieron en arrendamiento grandes extensiones de terreno ${ }^{13}$.

Con respecto a los primeros, la primera

11 Belza 1977, op. cit., pp. 249 y 250.

12 Barbería 1996, op. cit., pp. 37 y 123 y Cepparo 1987, op.cit., p. 42.

13 Ivanoff Wellmann 1997, op. cit, p.10 
noticia que se tiene de la llegada de colonos es en 1905 cuando grupos familiares arribaron a esta zona central de la Patagonia Chilena o Territorio de Aysén después de recorrer el oeste argentino desde el norte de la Patagonia. Al sentirse amenazados por las consecuencias que podían tener las campañas de ocupación de la Patagonia organizadas por Roca, y a la vez atraídos por la existencia de parajes deshabitados en el sur de Chile, decidieron instalarse en el sur. Transcurridos algunos años piden permiso de ocupación, que es concedido por la Oficina de Tierras y CoIonización de Punta Arenas en el año 1914. Pronto comienzan a llegar otros pobladores, aúnan sus esfuerzos y progresan económicamente. Sin embargo, debido al interés de grandes compañías que ya habían comenzado a explotar estas tierras, se lleva a remate los arrendamientos de los colonos libres, obligándolos a abandonar las tierras. Desde ese instante comienza a gestarse una historia de enfrentamientos y abusos de autoridad, conocida como Sucesos del Lago Buenos Aires o Guerra de Chile C hico, ocurridos en 1918 ... Conflictos donde están ligados los nombres de Braun y Menéndez Behety que conformaban el poderoso grupo magallánico que vio con preocupación que el floreciente grupo de pobladores del Lago representaban una amenaza para sus intereses en todo el territorio de Aysén ${ }^{14}$.

Precisamente, empresarios magallánicos, entre los que figuraban los mencionados, y Braun $\&$ Blanchard, a través de traspaso de derechos de ocupación de los primeros concesionarios, de la unificación de concesiones y de arrendamiento, se hicieron poseedores de dilatadas superficies en el Territorio de Aysén. Años más tarde aprovecharon la legislación argentina y arrendaron terrenos cercanos al lago Buenos Aires, en la zona del Deseado, que limitaban con los terrenos de las mismas sociedades del otro lado de la frontera. Sus intereses empresariales los llevaron a participar como socios principales de compañías o sociedades ganaderas, las que tuvieron más representatividad en tierras chilenas que en argentinas en esta latitud de la Patagonia. La zona norte de Santa Cruz dependió más de la inquietud pobladora y empeño de pobladores independientes mientras que la zona de Aysén, del interés especulativo de grandes empresas ganaderas.

Según la legislación chilena, las socieda- des estaban obligadas a instalar familias, construir caminos y establecer contactos marítimos con el resto del país. De allí que sólo tuvieron éxito las grandes compañías que contaban con una estructura organizativa y una infraestructura apropiada para realizar estas inversiones. Las deudas, la deficiente administración o la falta de cumplimiento de las obligaciones, provocaron el fin de algunas concesiones, aún de entidades integradas por importantes capitalistas ${ }^{15}$

Volviendo a la zona de Los Antiguos, también sucedió que. . . la mala elección del terreno, la falta de vías de comunicación, la deficiencia en las propagandas, las dificultades para concretar la compra de las tierras impidieron que las colonias creadas adquirieran el desarrollo deseado, sino que por el contrario vegetaron en tierras pobres, escasa la vegetación y perdidas en el desierto. No así las colonias bien ubicadas, donde el trabajo individual inmediatamente remunerado, transformó los campos yermos en hermosos establecimientos ganaderos ${ }^{16}$.

Eslo que ocurrió en las colonias Las Heras y Leandro N. Alem. Las buenas producciones agropecuarias de los arrendatarios de la zona norte de la provincia, impulsaron el surgimiento de centros de acopio y despacho de animales, lana y cuero, que también cumplían la función de abastecimiento y servicio básico de las áreas rurales cercanas. Los pueblos de Las Heras, Perito Moreno y Los Antiguos, tuvieron esos roles, los que favorecieron su poblamiento. Los buenos resultados de los primeros ganaderos motivaron la llegada de más interesados, aunque con menos recursos que los que ocuparon las zonas centro y sur de la provincia; y con la limitación de ocupar terrenos de dimensiones más reducidas que en el resto del territorio, menos de 10.000 ha.

Ese doble juego -menos capitales, más pobladores- motivó una mayor subdivisión de las propiedades, hecho que se manifestó en el significativo número de explotaciones agropecuarias en los departamentos del norte provincial: Deseado y Lago Buenos Aires. A pesar que el proceso provocó mayor cantidad de solicitantes de arrendamiento, no aumentó la población. La ganadería extensiva necesitaba grandes extensiones vacías y poca mano de obra. La consecuencia inmediata fue el subpoblamiento. Debieron pasar

15 Ibid., p. 13 y 22.

16 Carcamo 1972, op. cit., pp. 240-241. 
dos décadas para que se ocupara la mitad norte del territorio, de características más pobres que la meridional ${ }^{17}$.

Es importante hacer una pausa y resaltar que la organización del espacio patagónico, desde el punto de vista de la difusión de su poblamiento, está íntimamente relacionado con la historia de los movimientos migratorios y sus comportamientos particulares. Las actitudes y propósitos de los actores sociales de cada corriente de población, sus identidades culturales, sus capacidades económicas; y el momento en que actuaron, dejaron su impronta en el territorio ${ }^{18}$.

Así, el verdadero poblamiento de la localidad de Los Antiguos ${ }^{10}$ comenzó en la década de 1920 con el afincamiento de pobladores argentinos y españoles que, siguiendo la onda de difusión del este se instalaron en las márgenes del río Los Antiguos. Los primeros habitantes fueron atraídos por la posibilidad de explotar, como arrendatarios, tierras todavía libres de ocupación, a la vez alentados por el precio de la lana que había comenzado a subir. Como colonia pastoril, la zona de Los Antiguos se benefició con la entrega de lotes de 2.500 ha, no pudiendo concederse más de uno; y como colonia agrícola se subdividió en lotes de 100 ha, no pudiéndose entregar más de dos a una misma persona. La ventaja consistió en que hasta la quinta parte de las tierras podía adjudicarse gratuitamente a los primeros pobladores. ${ }^{20}$

Otro fuerte incentivo poblacional fue el excelente microclima que generaba el lago Buenos Aires. De allí que en 1924, el Ing. Agrónomo Tomás Davinson, al realizar una inspección a la Colonia Leandro N. Alem, aconsejó la reserva de estas tierras para la agricultura. Los pobladores supieron seguir esa sugerencia, ocupando primero las tierras en los alrededores de lo que sería el área nuclear del asentamiento en el que en 1928 se había instalado la escuela primaria y un destacamento policial; y estaban delineadas algunas chacras cuyos ocupantes cultivaban alfalfa ${ }^{21}$.

17 Cepparo 1987, op. cit., p. 42.

18 Cepparo 1997, op.cit., p. 343.

19 Este lugar ya tenía antecedentes de asentamiento preferido por los indígenas. Por sus especiales condiciones ambientales lo elegían como último reposo. Se lo llamaba I Keu Kenk = lugar de los ancianos o posada de los mayores.

20 Barbería 1996, op. cit., p. 114.

21 García 2000, op. cit., p. 659.
Durante los personales trabajos de campo realizados en los años 2000 y 2002, fueron encuestados algunos de los 37 primeros adjudicatarios registrados a fines de la década de 1930. Entre los que actualmente mantienen sus cultivos figuran: J acobo Erichsen, J uan, Luis y J osé B eroiza, J orge Seguel, Cristhian Treffinger y Oscar Funes. Sólo dos de estos pioneros continúan produciendo en las quintas originales. LoS demás compraron otras parcelas.

4.2 La delimitación del pueblo y los efectos de su posición

La traza urbana la realizó la policía en 1939, de acuerdo a las órdenes recibidas desde la G obernación del Territorio, respetando las construcciones existentes. Se deslindaron 16 manzanas para la planta de la futura ciudad donde las únicas edificaciones eran las de la escuela, el destacamento policial y la estación de radiocomunicaciones ${ }^{22}$. Servicios que manifestaban la función generalizada que tenía la mayoría de los asentamientos del área rural durante el período de auge de la explotación ganadera (1885-1930): lugar de servicios sociales básicos, de algunas instituciones gubernamentales y de las sucursales de los principales negocios de Punta Arenas, como la Sociedad Anónima Importadora y Exportadora de la Patagonia.

Esta era la principal compañía comercial de la Patagonia meridional, formada en 1908 por la fusión de las empresas de José Menéndez, Braun y Blanchard, ya mencionados. Contaba con sucursales en todos los puertos y pueblos del interior de Santa Cruz, cuyos servicios se extendían a sus áreas de influencia. Cubrían todos los rubros de importación, además de la exportación de lana, la representación de agencias internacionales de navegación, el otorgamiento de créditos, una flota mercante propia que monopolizaba el transporte entre Punta Arenas y Buenos Aires. El avance de estas empresas impidió el surgimiento y crecimiento del comercio local, especialmente en los reducidos y aislados poblados, siendo escasos los pequeños almacenes que funcionaban en los núcleos urbanos ${ }^{23}$.

Finalmente, la Dirección General de Tie-

22 Ibid.

23 Barbería 1996, op. cit., pp. 57 y 63; y Martinic 1976, op. cit. pp. 18-31. 
rras y Colonias recién decidió la creación oficial del pueblo de Los Antiguos, por decreto nacional 115.785 de septiembre de 1941, en los lotes $65,66,75$ y 76 de la sección II de la Colonia Leandro Alem. A pesar de las distancias que se debían recorrer y que los comienzos fueron muy modestos, los servicios que ofrecía el pueblo, aún siendo elementales, alentó a los pocos pobladores a permanecer en el lugar. ${ }^{24}$

Por otro lado, las comunicaciones largas, irregulares y en ocasiones interrumpidas por problemas climáticos; y el escaso desarrollo de la infraestructura caminera, provocaron graves problemas en su accesibilidad. La lejanía y la discontinuidad en los transportes elevaban los costos de la producción y en consecuencia limitaban la oferta de los bienes producidos en la zona a otros mercados, aún los más cercanos. Sin embargo, los beneficios del sitio y las experiencias favorables en los cultivos de alfalfa, legumbres, hortalizas y cereales, contribuyeron a subdividir las parcelas originales; y a extender y diversificar las plantaciones. ${ }^{25}$

Requiere un comentario especial la función que, desde 1914, tuvo el ferrocarril Puerto Deseado-Las Heras, aunque el proyecto original llegó a la mitad del recorrido planificado y el tráfico se detuvo a fines de la década de 1970. Para algunos historiadores patagónicos, el ferrocarril favoreció el crecimiento de un rosario de centros poblados a orillas de las vías. Provocó no sólo un gran movimiento comercial en las localidades mencionadas, sino en los asentamientos que estaban en sus áreas de influencia, entre ellos Los Antiguos. Mientras Puerto Deseado fue la puerta de salida de la producción regional, Las Heras se convirtió en punta de rieles y centro de acopio de la producción ganadera de las estancias vecinas y de las colonias establecidas en la zona cordillerana. Ambos fueron centros de distribución y abastecimiento de los productos manufacturados que llegaban en los mismos barcos que extraían la producción ${ }^{26}$.

Para otros autores, esta línea ferroviaria no favoreció a las poblaciones ni a sus zonas de influencia, ya que no brindó un servicio seguro, económico ni rápido. Los ganaderos preferían continuar transportando su producción, por sen-

24 Lenzi 1980, op. cit., p. 442.

25 Cepparo, 1983, op. cit., p. 18.

26 García 2000, op. cit., p. 640. das que ellos mismos habían trazado, al puerto de Comodoro Rivadavia ya que tenía la ventaja de ofrecer una mayor frecuencia marítima que Puerto Deseado, más capacidad de carga y experiencia en el traslado de las mercaderías desde este sector de la Patagonia ${ }^{27}$. Toda la producción regional se embarcaba desde este puerto con destino a Punta Arenas para su traslado a los mercados europeos. Cuando Punta Arenas perdió el liderazgo en el transporte naviero al surgir la posibilidad de trasladar las mercancías por el canal de Panamá, el comercio se hizo directamente desde los puertos de Santa Cruz por medio de firmas compradoras que las enviaban a Europa.

Un hecho destacado es que según las mercaderías que circulaban en el ferrocarril Puerto Deseado-Colonia Las Heras, y las transportadas a través de la ruta provincial № 520, hoy ruta provincial $\mathrm{N}$ o 43, el tráfico que iba desde la costa hacia el interior era mayor que el inverso ${ }^{28}$. Esta situación de desequilibrio entre la oferta y la demanda de mercaderías locales y extralocales que circulaban por las vías del norte provincial, hace pensar que la distancia actuaba de diferente manera según dónde se ubicaban los centros proveedores más importantes. Distancia y clima se potenciaban limitando la posibilidad del transporte debido a que aumentaban los costos de instalación de líneas y de mantenimiento de unidades y servicios en las zonas más alejadas. Hecho notable porque afectó la periodicidad, la cantidad de ofertas; y las demandas de servicios y mercaderías que surgían del noroeste de la provincia. En consecuencia impactó en la posición de la zona en los intercambios regionales y nacionales.

Es oportuno aclarar que, actualmente, el ferrocarril está inactivo y la salida de la producción agropecuaria ya no se realiza por $C$ omodoro Rivadavia, Puerto Deseado u otros puertos santacruceños, entre otros factores, por la competencia que significó el camión al utilizarse su servicio puerta a puerta para el traslado de la producción regional hacia los centros consumidores e industriales del norte del país. Los combustibles líquidos representaron durante un significativo lapso, las cargas que más movilizaron los puertos patagónicos. Fue el caso de Comodoro Rivadavia. Hoy la pesca se ha convertido

27 Barbería 1996, op. cit., p. 64.

28 Francini, Lorenzini et al (s/f), p. 143. 
en la actividad más dinámica de la costa debido al movimiento de fletes, de mano de obra y de capitales, reemplazando así la vitalidad que tuvo, primero, la actividad ganadera, luego la minera. La producción agrícola, por el contrario, no tiene el suficiente volumen de carga para que sea ventajosa su salida desde los puertos.

\subsection{Territorios marginales pero integrados}

L as referencias a las grandes distancias, el aislamiento, la escasa frecuencia de los transportes, nos llevan a retomar las consideraciones sobre áreas marginales mencionadas en las primeras páginas. Destaco que hay un grupo de autores que defienden un criterio de definición con el cual otros no coinciden. Por ejemplo, no siempre la marginalidad se manifiesta en los territorios alejados debido a que las relaciones económicas y los avances tecnológicos los han incorporado al mundo globalizado. Tampoco son los que tienen aptitudes físicas difíciles porque ecológicamente se los considera centrales o no afectados por la acción del hombre. Lo mismo sucede con los que están próximos a las regiones más integradas, pero socialmente pueden estar abandonados o excluidos por los organismos de decisión ${ }^{29}$.

Existen otras manifestaciones de marginalidad en las que los especialistas están de acuerdo. En general se refieren a aspectos socio-económicos entre los cuales resaltan: reducidos equipamientos, escasa disponibilidad de oportunidades, débil capacidad de innovación de los habitantes, notables carencias en las funciones urbanas y rurales. También mencionan los relacionados con las aptitudes físicas, la continua presión de los países desarrollados sobre las tierras y los recursos de los subdesarrollados; o los problemas de accesibilidad y circulación en espacios con potencialidades pero con altos costos para llegar a los mercados ${ }^{30}$. Desde cualquiera de las dos perspectivas, se advierte la fuerte presencia de estas características en la zona de estudio.

Paradójicamente, al volver al tema de la posición fronteriza de Los Antiguos y su impacto en el proceso de poblamiento, se comprenden los motivos que convirtieron al asentamiento en un lugar de paso para el tránsito de las mercan-

29 Leimgruber 1994, op. cit., pp.1-8. 30 Ibid. cías provenientes de Chile, especialmente desde las poblaciones que se habían instalado en los primeros años del siglo XX al sur del lago General Carrera en el territorio de Aysén, $C$ hile Chico entre ellas. Cruzaban plata y plomo que traían de poblaciones chilenas cercanas a la frontera; lana que iba destinada a los lavaderos de Alto Río Mayo en Chubut; madera, que era requerida en las localidades que estaban formándose al norte de Santa Cruz y sur de Chubut; ganado que era llevado por caminos que ellos mismos habían trazado hasta Comodoro Rivadavia, donde eran vendidos en la sucursal de La Anónima ${ }^{31}$. Y cuando el ferrocarril que nacía en Puerto Deseado llegó a Las Heras a mediados de la década de 1910, la producción de los ganaderos chilenos y argentinos se llevaba hacia esta última localidad y desde allí por ferrocarril hasta Puerto Deseado con destino final en los mercados europeos ${ }^{32}$.

Para los habitantes de la localidad chilena de Chile Chico, Los Antiguos siempre estaba en el recorrido de las mercaderías producidas en la región. Buscando la llegada a Santiago de Chile, eran trasladadas en un recorrido que incluía Los Antiguos, desde allí por la ruta nacional № 40 hacia el norte 0 por el camino de tierra hacia Las Heras en donde se trasbordaban al tren que las conducía a Puerto Deseado. En el puerto se embarcaban $y$, saliendo por el estrecho de Magallanes, llegaban hasta el centro de Chile.

La circulación de personas también fue constante, especialmente desde Chile hacia Argentina, por varios motivos. Primero, era frecuente el tránsito de ciudadanos chilenos que llegaban por territorio argentino desde la zona septentrional de su país o desde la zona de Coyhaique, para colonizar la región sur de Aysén, en la Patagonia chilena, como ya se mencionó ${ }^{33}$. Segundo, la mano de obra rural del área pastoril, y de las chacras o quintas, era en su mayoría de origen chileno y competía con otras comparsas de esquila, integradas por argentinos que venían todos los veranos desde el noreste del país. Tercero, cruzaban a nuestro territorio para trasladarse a otros lugares de Chile por medio de caminos que desde Perito Moreno se irradiaban hacia el norte y el sur debido a las difíciles comunicaciones que existían en la vertiente chilena. Intercambios, estos dos últimos, que aún continúan. Este

31 Ivanoff Wellmann 1997, op. cit., pp. 28-29.

32 Barbería 1996, op. cit., p. 63.

33 Ivanoff Wellmann 1997, op. cit., pp. 23-25. 
movimiento de mercaderías y personas motivó la construcción del resguardo aduanero Los Antiguos a comienzos de la década de $1940^{34}$.

El dinamismo que generaba la función de corredor de paso de mercaderías producidas en Chile, benefició a la población argentina con crecimiento poblacional y económico. Función que tuvo algunos altibajos que iban a la par de las relaciones bilaterales. El patrón de articulación binacional generó crecimiento demográfico y movimientos económicos en el poblado, pero abruptamente en 1955 leyes de orden nacional pusieron fin a este esquema económico llegándose a suspender el tránsito comercial ${ }^{35}$. Pero también fueron esas mismas circunstancias las que motivaron a la población la valorización de las posibilidades que ofrecían el microclima y el suelo del valle para los cultivos intensivos. En los momentos en los que se detuvo la circulación entre los dos países, Los Antiguos continuó con sus funciones comerciales, de servicio básico y de centro de aprovisionamiento del área rural circundante.

Actualmente, las relaciones son muy dinámicas. Durante el ciclo productivo de la cereza, especialmente durante la cosecha de la fruta, pobladores de Chile $\mathrm{C}$ hico representan la fuerza de trabajo más numerosa. Un promedio de 200 personas, desde adolescentes hasta ancianos, participa anualmente en las labores de cosecha, clasificación y empaque. De la totalidad de personal transitorio, el $75 \%$ proviene de Chile Chico. Un puente cruza desde hace pocos años el río J einimeni y transportes especiales los trasladan todos los días a su lugar de origen a menos de 10 $\mathrm{km}$ de distancia ${ }^{36}$.

Según el Subsecretario de Trabajo de Santa Cruz, la mano de obra chilena que llega para la cosecha es atraída por la posibilidad de trabajo pero más aún por los beneficios del cambio de moneda. Una buena zafra por año puede representar una producción aproximada de 250 toneladas y requiere de una contratación temporaria de 800 personas que deben seleccionar y manipular cuidadosamente la fruta. Para cosechar alrededor de mil plantas de cereza se

34 Ibid. y Palma Godoy 1999, op. cit., p. 189. Las encuestas y entrevistas personales realizadas a lo largo del trabajo en la zona de estudio, han permitido reunir esa misma información.

35 Palma Godoy 1999, op. cit., p. 189.

36 Cepparo 2005. necesitan entre 25 y 35 zafreros con una paga por kilogramo de fruta recogida entre 0,30 y 0,35 centavos. En una jornada laboral de un día, un buen cosechador llega a juntar cerca de 150 kilogramos y con la paga mínima llega a 45 pesos por día, que para los chilenos, por razones del cambio, les rinde el doble en su país ${ }^{37}$.

4.4 Los Antiguos y la organización políticoadministrativa de la zona norte de Santa Cruz

Regresando al tema del proceso de poblamiento de Los Antiguos, resalto que la concentración de población siguió siendo muy pequeña. Sin embargo, en 1948 se conformó la primera Comisión de Fomento durante la Gobernación Militar de Comodoro Rivadavia. Esta situación conduce a la referencia de las modificaciones que Santa Cruz experimentó en sus límites administrativos en la década del 40 y que indirectamente afectó a la organización políticoadministrativa de sus poblaciones. El Gobierno Militar del General P. Ramírez estableció por decreto 13.941 del 31 de mayo de 1944, la Zona Militar de Comodoro Rivadavia que dependía del Ministerio de Guerra y comprendía la zona de explotación petrolífera de Comodoro Rivadavia ${ }^{38}$. La medida tenía como antecedente la presencia de las unidades militares que formaban la Agrupación Patagonia, basada en la necesidad de mantener la seguridad de la región.

Sucesivos decretos fueron ampliando los límites de la gobernación que, desde el Atlántico a la Cordillera, integraba a las poblaciones ubicadas desde el paralelo que pasa al norte de Camarones hasta el río Deseado al sur. Durante un lapso de 11 años, 1944-1955, los pobladores tuvieron un régimen político-administrativo diferente: el gobernador nombrado por el Poder Ejecutivo se manejaba, a diferencia del resto de la Patagonia, con razonables recursos presupuestarios, con flexibilidad de movimientos; designaba los Comisionados Municipales, las J untas de Fomento, los J ueces de Paz, y actuaba como J efe de Policía del lugar ${ }^{39}$.

El sistema de las J untas de Fomento so-

37 TIEMPO SUR, 2001. «Preocupa la contratación de chicos chilenos para la zafra de la fruta fina» Río Gallegos, 30 de junio. En: www.tiemposur.com

38 Boletín O ficial 5-6-44.

39 Hudson 2000, op. cit, p. 568 y Zampini 1975, op. cit., pp. 93 y 94 . 
lucionó los problemas relacionados con las atenciones básicas de la población y con los servicios públicos de los pequeños poblados cuyo número de habitantes era menor al mínimo establecido para tener gobierno propio. De esta forma, la población de Los Antiguos mantuvo las mismas facultades que otros pequeños centros de la provincia que tenían más de 500 habitantes y menos de 1000 concentrados en una superficie de 2500 hectáreas. ${ }^{40}$

Cuando la Ley 14.315 de 1954 derogó la Ley Orgánica de los Territorios Nacionales de 1884 y sancionó la Provincialización ${ }^{41}$, estableció el régimen municipal en todas las localidades aún en las escasamente pobladas. De allí que las localidades con más de 500 empadronados tendrían derecho a Municipio, las de menos de 500 habitantes tendrían Comisiones de Fomento ${ }^{42}$. Recién el 22 de febrero de 1970, por decreto 1.258, se declaró Municipalidad a Los Antiguos, según lo que establecía la Constitución de la Provincia de Santa Cruz en su artículo № 138, en cada centro poblado que cuente con un número mínimo de 1000 habitantes, se constituirá un municipio encargado de la administración de los intereses locales ${ }^{43}$. Durante esa década se subdividieron las manzanas de su zona urbana con la edificación de casas realizadas por medio de un plan provincial de viviendas. Este incentivo fortaleció el poblamiento de la zona urbana ${ }^{44}$, al mismo tiempo comenzó la formación de la estructura territorial en la zona agraria.

5. LOS ANTIGUOS Y EL CRECIMIENTO DE LA POBLACIÓN Y LA TENDENCIA A LAS ACTIVIDADES TERCIARIAS EN EL MARCO DEL MODELO PROVINCIAL

Recién a partir de 1947 la población de Los Antiguos comenzó a registrarse en los Censos Nacionales entre los asentamientos con menos de 500 pobladores. En esa fecha tenía 388 habitantes. A lo largo de los censos esta localidad muestra un crecimiento moderado y muy

40 Hudson 2000, op. cit., p. 560.

41 Anales de Legislación Argentina. T.XIX - A, Año 1954, Leyes 14.298-14.400, p. 91.

42 Hudson 2000, op. cit., p. 570.

43 Honorable Convención Constituyente. Provincia de Santa Cruz. 1982. Constitución Provincial. Artículo 138. En: Boletín e Imprenta Oficial, Río Gallegos.

44 García 2000, op. cit., p 660. pausado al igual que la mayoría de las poblaciones ubicadas en las áreas centrales y occidentales de la provincia y a pesar de los aumentos poblacionales que experimentó la provincia. En el perío do 1960-1970, Santa Cruz vivió un notable crecimiento coincidente con una de las más fructíferas políticas de promoción patagónicas. Sin embargo, en la década siguiente la caída poblacional fue semejante a la que se produjo después de la década de 1930. Circunstancia que reflejaba los impactos provocados por las recurrentes crisis provinciales y por la falta de continuidad de una política de estado en beneficio de las economías regionales o extrapampeanas. En el caso de Santa Cruz, las localidades del interior tenían como marco las extensas superficies que trabajaban al límite de su rentabilidad o que comenzaban a ser abandonadas debido a las consecuencias que provocaban los altos índices de desertificación en el área de las mesetas.

El problema ganadero aumentó la expulsión de población del ámbito rural y la selectividad de la mano de obra con el consiguiente incremento de los índices de masculinidad. Combinado con los conflictos económicos nacionales, disminuyó la llegada de inmigraciones afectando el crecimiento vegetativo, la densidad poblacional y el proceso de urbanización, sobre todo en las zonas más alejadas. La demora en la ocupación del centro urbano de Los Antiguos es un reflejo de esta situación.

La distribución de la población, sumamente irregular, a lo largo de todos los censos, manifiesta la mayor concentración en el departamento de Güer Aike, en el sur de la provincia donde se ubica la capital con el mayor peso poblacional, casi 6.000 habitantes en 1947 y más de 78.000 en 2001. Le seguía el departamento de Deseado con la localidad de Puerto Deseado que tenía más de 3.000 habitantes en el primer censo considerado, posteriormente reemplazada por Caleta Olivia, que mantuvo el segundo puesto en cantidad de habitantes a partir de 1960 hasta la actualidad.

Los asentamientos de los departamentos de Güer Aike y Deseado concentran aproximadamente el $80 \%$ de los habitantes de la provincia, con la ventaja de localizarse, la mayoría, a lo largo de los $1000 \mathrm{~km}$ de costa marítima que tiene la provincia, pero sólo tres de ellos superan los 10.000 hab. Río Gallegos y Caleta Olivia son cabeceras de los departamentos mencionados, centros administrativos y de servicios; núcleos que 
apoyan a la actividad petro-gasífera, ganadera y pesquera; además de haber sido asiento de varias agrupaciones de las Fuerzas Armadas de la Nación. Todo ello contribuyó al significativo crecimiento demográfico en contraposición a las demás localidades provinciales. La explotación de hidrocarburos que, desde 1940, se extendió por la mayoría de los pueblos del norte de Santa Cruz, y desde Río Gallegos hacia el sur del área continental, influyó positivamente en el poblamiento de los departamentos de Deseado y de Güer Aike dando trabajo a los habitantes y atrayendo un importante número de mano de obra de otros lugares del país.

Las cifras censales reflejan con claridad la debilidad estructural demográfica de la mayoría de los centros urbanos del interior de la provincia. Además de las limitaciones mencionadas, los asentamientos santacruceños se perjudicaron con el cambio de modelo económicos desde fines de la década de 1980. La privatización de las empresas nacionales, Yacimientos Petrolíferos Fiscales y Yacimientos $C$ arboníferos Fiscales, provocó la retirada de gran cantidad de empleados públicos, además de la población perteneciente a las Fuerzas Armadas.

Por otro lado, en el ámbito rural, se acentuaba la crisis ganadera y faltaban paliativos en el campo; y en los medios urbanos se diversificaban y concentraban más empleados en los servicios básicos comunitarios, salud, seguridad y educación. Si bien son actividades que generaron nuevas fuentes de trabajo, muchas veces eran ficticias porque la desocupación estaba encubierta por el mismo Estado. En efecto, los pobladores que se dedican a los servicios, por ejemplo, en Los Antiguos, Perito Moreno y Gobernador Gregores, superan a los que se relacionan con las actividades primarias y genuinamente productivas, la fruti-hortícola en la primera localidad y los cultivos de forestales y de alfalfa en las otras.

No está de más resaltar que si se considera el total de las actividades agrupadas en comercio, transporte y servicios, el grado de terciarización en la provincia era y es relevante. En 1980, ascendía al 47,6\%, y en 1991 al $69,8 \% 45$. Los datos por grupos de actividades en Santa Cruz según el último Censo de Población

45 Estos datos como otros que figuran en el texto son de elaboración propia sobre la base de: González Pedemonte, Lafuente, Cepparo et al 1985, op. cit., p. 19 y 20. de 2001 no han sido publicados todavía por el INDEC. Sin embargo, la información existente demuestra que, a lo largo de los últimos 10 años, la cantidad de empleados en el sector público ha predominado sobre los del sector privado. En el año 2001, el 43\% de la mano de obra ocupada estaba ubicada en la administración pública, el $38 \%$ en el ámbito privado y sólo el $13 \%$ estaba integrado por trabajadores por cuenta propia. La diferencia es más notable en las pequeñas localidades santacruceñas ${ }^{46}$. Es el caso de Los Antiguos donde, en el año 2001, el 63\% de su población dependía de los organismos del estado y los servicios públicos, $15 \%$ estaba relacionada con emprendimientos privados y el $16 \%$ era cuentapropista 47.

Actualmente, la categoría urbana de Los Antiguos, cuya población de 2.047 habitantes representa el $1,03 \%$ del total provincial, es la de un centro local secundario ${ }^{48}$. Se ubica en una posición elemental por la cantidad de población que posee, por el carácter básico de las funciones urbanas al servicio de las zonas rurales inmediatas, y porque presenta una considerable dependencia de un núcleo mayor, Caleta Olivia que actúa como centro regional secundario. Ambos están ubicados dentro del área de influencia del gran centro de servicios de la Patagonia Central, Comodoro Rivadavia, y no de Río Gallegos, capital de la provincia.

Ya se consideraron los rasgos de marginalidad que presenta Los Antiguos, pero la escasez de servicios que ofrece permite, ahora, relacionar esta situación con las características de las áreas escasamente pobladas. Los especialistas en el tema coinciden que son espacios aislados con escaso crecimiento poblacional, débil cantidad y diversificación de servicios y escasas innovaciones en el ámbito urbano y rural. En cuanto a las áreas rurales poco pobladas, la indiferencia hacia las actividades productivas, ha motivado que las denominaran espacios paralizados o quietos. Afirmaciones muy contundentes que constituyen una fuerte incitación para verificar si estas desfavorables situaciones se repiten en Los Antiguos y en su área de cultivo.

Por eso se hace referencia al equipa-

46 REPÚBLICA ARGENTINA. INSTITUTO NACIONAL DE ESTADÍSTICAS Y CENSOS. Censo Nacional de Población, Familias y Viviendas 2001. Cuadro 2.11.

47 Ibid.

48 Cáceres 2000, op. cit., p. 893. 
miento que ofrece la ciudad. Comprende un escaso comercio de detalle: carnicería, zapatería, farmacia, almacén de ramos generales, estación de servicio. Existen cuatro establecimientos industriales, 22 dedicados al comercio y 13 relacionados con los servicios. Mientras que de los primeros no se cuenta con datos con respecto a la mano de obra que ocupan, los segundos representan el $14 \%$ de los puestos de trabajo; y los terceros, el $42 \%{ }^{49}$.

La escolaridad está representada por un establecimiento de nivel inicial, dos de nivel primario y EGB, una escuela primaria para adultos, uno de nivel medio y polimodal y uno de nivel medio para adultos. Gimnasios y centros culturales que dependen del municipio completan las prácticas culturales y educativas. Un hospital de baja complejidad, donde no se practican cirugías, está a cargo del servicio asistencial; y una sucursal del Banco Provincia resuelve los trámites financieros. D os hoteles y algunas cabañas cubren las necesidades de hospedaje de los viajantes, turistas o visitantes que llegan a la localidad por la ruta provincial $\mathrm{N}$ o 43, asfaltada; la nacional $\mathrm{N}$ o 40 , de ripio; la provincial № 45 , de ripio, que llega hasta el paraje Ingeniero Palavicini en el sur del asentamiento; y la ruta nacional chilena $\mathrm{N}$ o 7, de ripio, que une a Los Antiguos con Chile Chico. La zona de quintas posee los servicios básicos de energía eléctrica, de gas envasado transportado desde Comodoro Rivadavia y telefonía fija y móvil. Y con respecto a las telecomunicaciones, no existe ningún nodo local de acceso a Internet, por lo tanto el ingreso a ese servicio se limita a conexiones particulares o a través de la línea telefónica de larga distancia ${ }^{50}$.

De modo que, en la escala urbana, la cantidad y calidad de servicios de Los Antiguos demuestran la correspondencia con los rasgos de las áreas escasamente pobladas. Pero, en el ámbito agrario, el avance lento pero constante de las plantaciones y la disminución paulatina de las superficies incultas, reflejan que es un espacio cambiante. Características que alejan estas superficies cultivadas de los conceptos negativos que tienen los espacios agrarios escasamente poblados, quietos o paralizados, como algunos auto-

49 SANTA CRUZ. DIRECCIÓN GENERAL DE ESTADÍSTICAS Y CENSOS. 2000. Síntesis de los Indicadores Socioeconómicos y de Infraestructura, pp. $1-5$.

50 lbid. res los califican.

Sin embargo, sería una postura explicativa muy ligera no tener en cuenta la capacidad de innovación de los actores sociales locales y la energía con la que llevan a cabo sus inquietudes. Innovaciones que recién ahora han comenzado a visualizarse en las actitudes de los productores frutícolas. Un ejemplo de ello es la inversión de los excedentes, generados en los últimos años, en la tecnología que se necesita en cada uno de los eslabones del circuito productivo de la cereza. Pero simultáneamente también está presente la pesada influencia de los rasgos culturales de la comunidad, acostumbrada a depender de los empleos públicos o de los subsidios ganaderos.

\section{CONCLUSIONES}

Desde el punto de vista conceptual, se han transferido a este trabajo las ideas de Chapman y Leimgruber. El primero consideró que las propiedades del medio físico, indudablemente, afectan de manera significativa los esquemas espaciales de la actividad humana ya que pueden actuar como una restricción o como una oportunidad para el desarrollo de los mismos. Sin embargo, enfatizó mucho más el rol de la evolución de los procesos económicos y culturales ya que influyen sobre las decisiones para localizar una actividad o establecer un determinado uso del suelo.

Así, se describieron y explicaron los hechos relacionados con la expansión del poblamiento de Los Antiguos y sus actividades; y los intercambios entre las comunidades fronterizas. Procesos relacionados con intereses que variaron con el tiempo, con iniciativas ventajosas en sus inicios pero conflictivas posteriormente, con la continuidad o discontinuidad de las funciones y de las fuerzas que guiaron los sucesos. Sucesos que manifestaron diferentes estructuras sociales pero que en definitiva los condujeron a su articulación y a su identificación como territorios marginales pero integrados. De ese modo, según nuestro criterio, se ha logrado superar el tradicional tratamiento dicotómico, ambiente-sociedad, y se ha enriquecido la visión del accionar de los agentes sociales en un territorio distante y con limitaciones naturales y culturales como los de la Patagonia Meridional.

Sobre la base de las ideas del segundo de los autores, se hizo referencia al aislamiento y la inaccesibilidad de las zonas marginales. Las 
características de la zona de estudio demostraron que: 1. existen situaciones que quiebran la relación directa entre alejamiento y marginalidad cuando interviene un factor con más fuerza como es la necesidad de integración; y 2. aún hoy los considerables trayectos que separan a las poblaciones, continúan teniendo un fuerte impacto a pesar del avance tecnológico de los medios de comunicación. Estos relativizan las distancias absolutas pero no las logran vencer definitivamente. Impacto que no hace más que confirmar la influencia que tienen los problemas de accesibilidad y circulación sobre los territorios aislados y lejanos.

En efecto, a medida que se desarrolló el tema de la posición periférica de Los Antiguos, de las relaciones con Chile Chico, de las distancias a los medianos y grandes centros urbanos, de los inconvenientes en la circulación, de los costos de los transportes, de las limitaciones ambientales y los elementales servicios urbanos, se fue advirtiendo la fuerte presencia de las características de marginalidad en el noroeste de Santa Cruz.

Pero también se ha detectado que es un caso ejemplificador con respecto a la paradoja que un mismo lugar presente manifestaciones de marginalidad y centralidad. Así, frente a la ausencia o debilidad de las vías de comunicación en los territorios vecinos de la región de Aysén, se magnificó la función que tienen los caminos del norte santacruceño y se valoró la posición de Los Antiguos como asentamiento fronterizo con roles de acceso, posada y descanso, de servicio y aprovisionamiento básico. De hecho Los Antiguos fue un lugar de paso esencial de personas y mercaderías entre el norte y el sur de Chile, por lo tanto muy apreciado por los habitantes de la Patagonia chilena central.

Por otro lado, el crecimiento de la población de Los Antiguos y su orientación a las actividades terciarias, refleja la fuerte influencia del modelo provincial donde el Estado es el principal empleador. Sin embargo, los últimos datos registrados, demuestran el rol que ha comenzado a tener el sector primario en la localidad. La fruticultura intensiva, el agroturismo y la producción de pasturas para corte, son las actividades que, es de esperar, continúen modificando las preferencias laborales de los trabajadores independientes, es decir los motiven a dejar los servicios y orientarse a actividades identificadas con los recursos naturales. El fuerte rol que el Estado siempre ejerció en Santa Cruz, y la política recurrente a no impulsar las actividades genuinamente productivas, contribuyen a justificar las debilidades y lentitudes en el dinamismo rural y en el equipamiento urbano.

Por fin y a pesar de la idea generalizada que los efectos de la globalización han beneficiado aún a los territorios marginales, se considera que existen ciertas manifestaciones cuyo grado de influencia supera el peso que ejerce el sistema global. Esas expresiones, reflejadas con mayor y menor intensidad en el área de estudio, son:

1. la distancia relativa respecto a los servicios básicos, que provoca la carencia de innovaciones o la lentitud para que ellas influyan;

2. el abandono o la exclusión por parte de los organismos de decisión que detienen o demoran la expansión de los servicios y acrecienten los sentimientos de olvido y desamparo que experimentan las zonas más alejadas;

3. las dificultades en la accesibilidad y la circulación; y los altos costos que representa vencerlas;

4. la falta de identidad con respecto a los recursos locales generando desinterés por las actividades genuinamente productivos.

Sin embargo, también se rescata el valor que tienen los vínculos entre los territorios y para ello recordamos las palabras de Roccatagliata cuando dice que la estrategia en la Patagonia es la complementación y articulación de las subregiones. Para ello son importantes proyectos de desarrollo y ordenamiento territorial basados en inversiones escalonadas y a largo plazo ${ }^{51}$. De ese modo, según nuestro criterio, aún cuando estén en posiciones periféricas y distantes de los centros más dinámicos, pueden consolidarse y alejarse de ciertos rasgos de marginalidad.

\section{BIBLIOGRAFÍA}

ANALES DE LEGISLACIÓN ARGENTINA.T.XIX - A, 1954. Leyes 14.298-14.400.

ANDREOLI, M., BRUNORI, G.L., TELLARINI, V., 1989. I sistemi agricoli in aree marginali. Mencionado por Leimgruber, W., 1994. Marginality and marginal regions: problems and definition. En: Chang-Yi Davis Chang (ed). Marginality and development issues in marginal regions, Taipei, National Taiwan University.

51 Roccatagliata 1987, op. cit., p. 94. 
BARBERÍA, E., 1996. Los dueños de la tierra en la Patagonia Austral. 1880-1920. Santa Cruz, Universidad Nacional de la Patagonia Austral.

BELZA, J .E., 1977. En la isla del fuego, T.3, Buenos Aires, Publicaciones del Instituto de Investigaciones Históricas de Tierra del Fuego.

CÁCERES, A., 2000. G eografía Urbana: Estructura y paisajes urbanos de Santa Cruz. En: El Gran Libro de Santa Cruz. T. 2, Madrid, Milenio Ed.

CARCAMO, M., 1972. Evolución histórica del régimen de la tierra pública. 1810-1916, Buenos Aires, Eudeba.

CEPPARO DE GROSSO, M. E., 1987. Confrontación de las redes de transporte entre la faja fronteriza santacruceña y la chilena contigua. Revista Waxen (2) Río Gallegos, Instituto Universitario de Santa Cruz.

CEPPARO DE GROSSO, M.E., 1993-94. Hacia la búsqueda de relaciones más complejas entre las redes y los asentamientos de dos espacios australes fronterizos. Anales del Instituto de la Patagonia 22:77-90. Punta Arenas, Universidad de Magallanes.

CEPPARO DE GROSSO, M.E., 1997. Las modalidades de ocupación de la Patagonia según la difusión de las ondas de poblamiento. 1885-1925. En: Revista de Historia Americana y Argentina XIX (37), Mendoza, Fac. de Filosofía y Letras, UN de Cuyo.

CEPPARO DE GROSSO, M.E., 2000. Desequilibrios y expectativas. Convivencia de dos procesos productivos de base agraria en la provincia de Santa Cruz. En: $C D$ Rom del II Encuentro Internacional Humboldt. Periferias, Regiones y Países. Mar del Plata.

CEPPARO DE GROSSO, M.E., 2005. Incipiente circuito productivo en una pequeña sociedad de la Patagonia Meridional Argentina. En prensa.

CHAPMAN, K., 1979. People, pattern and process. London, Arnold.

FRANCINI, E., LORENZINI, H., ROMERO, S., CURTO DE CASAS, S. (s/f). La función nodal de Comodoro Rivadavia en la circulación patagónica. Boletín GAEA, Comodoro Rivadavia.

FURLANI DE CIVIT, M.E., 1991. Las comunicaciones en las provincias de la frontera oeste argentina. Boletín de Estudios Geográficos XXIV (87), Fac. de Filosofía y Letras, U.N. de Cuyo.

GARCÍA, A., 2000. El origen de las localidades de Santa
Cruz y algunos aspectos de su desarrollo. En: EI Gran Libro de Santa Cruz. T.2, Madrid, Milenio Ed.

GONZÁLEZ PEDEMONTE, U., LAFUENTE, H., CEPPARO DE GROSSO, M.E., CALDERÓN, M., LUCHETTI, G. Y PAZ, C., 1985. Centros de Servicios Rurales de Santa Cruz. Inédito.

GUTIERREZ, M.J ., FURLANI, M.E., 1993. Geografía Agraria. Buenos Aires, Ceyne.

HONORABLE CONVENCIÓN CONSTITUYENTE. Provincia de Santa Cruz. 1982. Constitución Provincial. Artículo 138. En: Boletín e Imprenta Oficial, Río Gallegos.

HUDSON, M., 2000. La política en Santa Cruz (1884-1955). Lineamientos y problemáticas. En: EI Gran Libro de Santa Cruz. Madrid. Milenio Ed.

IVANOFF WELLMANN, D., 1997. La Guerra de Chile Chico o "Los sucesos del lago Buenos Aires", Valdivia, Chile, Tecnoimprenta Color Ltda.

LEIMGRUBER, W., 1994. Marginality and marginal regions: problems and definition. En: Chang-Yi Davis Chang (ed). Marginality and development issues in marginal regions, Taipei, National Taiwan University.

MARTINIC BEROS, M., 1976. La expansión económica de Punta Arenas sobre los territorios argentinos de la Patagonia y Tierra del Fuego, 1885-1925. Anales del Instituto de la Patagonia 7:5-42, Punta Arenas.

LENZI, H., 1980. Historia de Santa Cruz. Buenos Aires, Lomelino Hnos.

PALMA GODOY, M., 1999. La cultura de la cereza como factor de desarrollo local. El caso del valle de Los Antiguos. La Patagonia de los noventa: sectores que ganan, sociedades que pierden. SALVIA, A. (comp.), Buenos Aires, Ed. Colmene.

REPÚBLICA ARGENTINA. INSTITUTO NACIONAL DE ESTADÍSTICAS Y CENSOS. 2001. Censo Nacional de Población, Familias y Viviendas Cuadro 2.11.

SANTA CRUZ. DIRECCIÓN GENERAL DE ESTADÍSTICAS Y CENSOS. 2000. Síntesis de los Indicadores Socioeconómicos y de Infraestructura.

SCHMIDT, M., 1998. An integrated systemic approach to marginal regions: from definition to development policies. Perceptions of Marginality J ussila, $H$., Leimgruber, W. y Majoral, R. (ed). England, Ashgate.

ZAMPINI, V., 1975. Chubut. Breve historia de una provincia argentina, Gaiman, Chubut, El Regional. 